\title{
Segmenting the Optic Disc in Retinal Images using Thresholding
}

\author{
Prashant Choukikar \\ Department of Electronics \& \\ Communication \\ SISTEC Bhopal India
}

\author{
Arun Kumar Patel \\ Department of Electronics \& \\ Communication \\ SISTEC Bhopal India
}

\author{
Ravi Shankar Mishra \\ Department of Electronics \& \\ Communication \\ SISTEC Bhopal India
}

\begin{abstract}
Segmentation of an optic disk location is a very essential task for retinal image analysis. Most of the pervious method uses the principal component analysis method, which are computationally complex. This paper presents a simple and semi automatic approach for segmentation of the optic disc in retinal images using thresholding and boundary extraction. First, the true colour Retinal images are converted to gray image and then image is enhanced using histogram equalization. This improves the efficiency of the conventional thresholding method. The proposed method of segmentation utilizes gray level thresholding for segmentation and then morphological operators are used for extracting the optical disk. The method is tested on the different optical disc images. The method is less complex and efficiently recognizes the boundary of the optic disc and also evaluates the optical canter. The optical canters are tabulated for original and corrupted images.
\end{abstract}

\section{General Terms}

Retina, Fundus, Image Processing, Morphology

\section{Keywords}

Optical Disc Detection, Segmentation, Histogram Equalization, Erosion, Dilation, thresholding,

\section{INTRODUCTION}

Identification of eye disease through analysis of retinal images has received the attention of many researchers. Detection of the optic disc (OD) is most essential for the automatic analysis of digital colour retinal images [1, 2 and 14]. Since the optical disc is different for healthy and corrupted eyes. It facilitates the tracking of various anatomical features and also in the extraction of Exudates, Drusens and locating the macula.etc present in the retina of human eye. The fundus includes the retina, the optic disc, and the macula. These structures are shown in Figure 1.

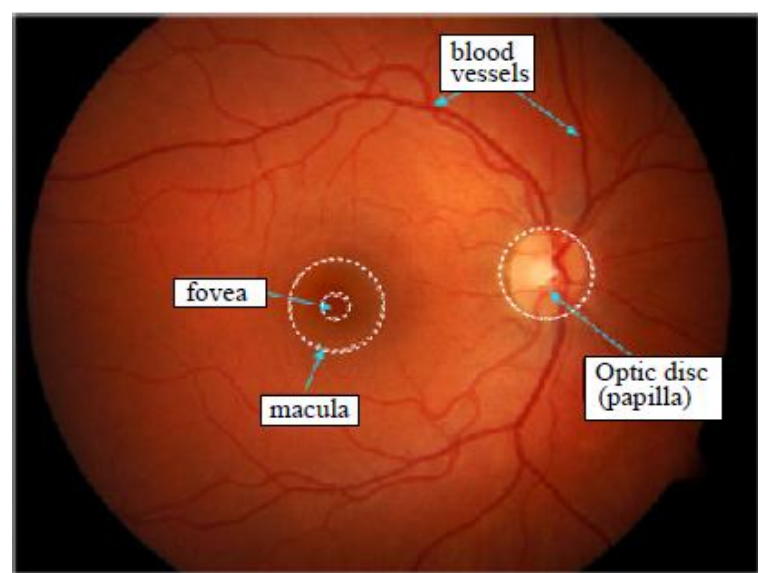

Figure 1 Structure of the fundus retinal image
Retina is the largest part of the fundus which is the interior surface of the eye. The optic disk is the brightest part in the normal fundus image that can be seen as a pale, round or vertically slightly oval shape disk $[7,13]$. This is the entrance area of blood vessels and optic nerves to the retina human eyes. The localization of the optic disc is critical in retinal image analysis for measuring distance and identifies other anatomical parts in retinal images. Pathology on or near the optic disc can have a more severe effect in vision.

Basically any method of optical disc location is based on the three essential stages. Figure 2 shows the basic processing steps used for optical disc location. Various methods have been designed by modifying these stages. The pre processing stage is used to enhance the appearance of the retinal images. The most common way of pre-processing is to use filters for noise removal and contrast enhancement for visual quality improvement.

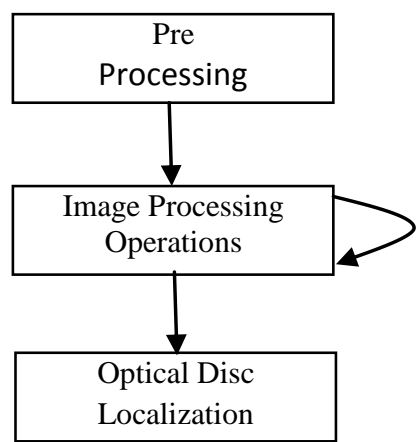

Figure 2 Basic process of optical disc location

After enhancing the image quality desired objects are extracted using segmentation operation. Segmentation is typically associated with pattern recognition problems. Image processing stage segments the various sections of the retinal images. The third stage uses features (shape, size, brightness) of the optic disc for identification of the optic disc in a normal retinal image. The circle fitting algorithms are widely used in this context. The efficiency of the thresholding based optical disc localization technique mainly depends upon the efficiency of the enhancement stage and on the efficiency of thresholding stage. Thus it needs to improve the enhancement quality of the images.

Two basic approaches used for finding the optical disk are Model based approach [11, 12] and Image feature based approach [5, 6]. In model based approaches models the geometrical directional pattern of the retinal vascular system. It implicitly embeds the information on optical disc as a point of convergence of all vessels. In order to find optical disk feature based approach uses the relative high brightness of optic disk, and the round shape of optical disc as compared to the rest of the fundus image. The problem of optic disc 
detection has rarely received unique attention. In this paper proposed a robust method for locating the optical disc and its canter. Section 2 has discussed some of the related existing works. The section 3 describes the proposed optical disk segmentation method using multilevel thresholding and morphological operators. The next section presents the results based on proposed method. It is found that this method is computationally less complex and efficient

The proposed communication system block diagram is explained in the section 4. The LMS and RLS linear equalizers are described in the section 5. The results of performance evaluation are given in the section 6 followed by the conclusion in section 7 .

\section{LITERATURE REVIEW}

There is lot of research done for the improvement of optical image quality as [4, 9, 12, and 14]. Contrast limited adaptive histogram equalization (CLAHE) method by Etta D, Pisano [5] were proposed to improve the image contrast for medical image applications and to overcome the amplification of noise problem. Hitam et al. [9] have presented a combination of the CHAHE in RGB and HSV colour spaces for underwater images. Agung W et al.[4] have proposed to use the CLAHE method for enhancing the colour retinal images using the (green) $\mathrm{G}$ channel. In the colour retinal image a unique characteristic exist than other medical images that these images in green $(\mathrm{G})$ channel, the blood vessels looks more contrast than the background.

The principal component analysis (PCA) is commonly used for optical disk analysis. Huiqi Li and Opus Chutatape [6] in 2001 proposed to automatically locate optic disk in retinal images. The desired regions in the intensity images are first determined by clustering the brightest pixels. Principal component analysis (PCA) is then applied to these candidate regions. The minimum distance between the original retinal image and its projection onto "disk space" is located as the centre of optic disk. But method was time consuming. Niemeijer [11] et al. in 2004 used 100 images for the comparative study of the retinal vessel segmentation method using model based approach, on new publically available database. Foracchia et al. [12], in 2000 uses matched filter response for detecting the vessel calibre irregularities used the 20 image subset. P. C. Siddalingaswamy and G. k prabbu [7] in 2007 presented an algorithm for the localization and segmentation of optic disk and fovea. Optical disk localization is achieved by iterative threshold method. D. Santhi et al. [13] in 2007, proposed the method of locating the optical disc in retinal images. At pre processing stage histogram equalization is used and the local contrast is enhanced in HIS colour space then for segmentation Otsu thresholding method along with multi level thresholding is used. Method was little complex. Qinghui et al. [14] have used vascular direction and intensity variance for finding the horizontal and vertical locations of the optical disc. The method seems bit fuzzy and complex. Other efficient retinal image segmentation methods are [15 and 16].

\section{PROPOSED SEGMENTATION OF OPTICAL DICS}

In this paper a post image processing method is proposed in which firstly the input image is equalized and then histogram based multilevel thresholding is applied on it. In the next stage the morphological operator's erosion and dilation are used for the optical disk identification. The block diagram of proposed method is shown in Figure 3. The procedure is basically having two stages Viz. Image enhancement and Disc segmentation.
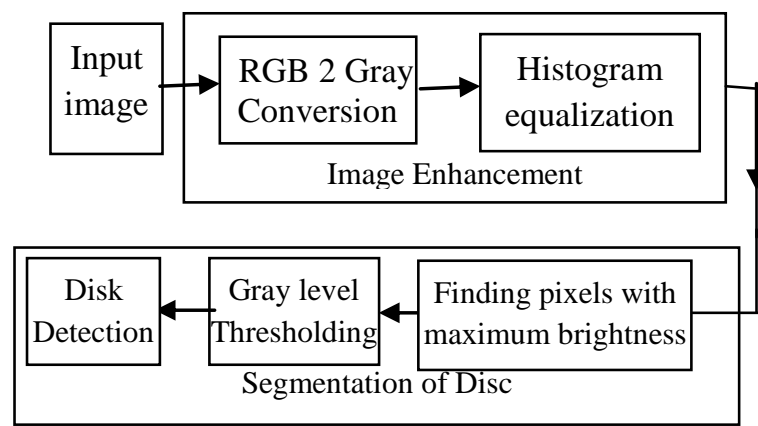

Figure 3 Block diagram of proposed method

\subsection{Gray image conversion}

Colours displayed on retinal images are usually defined in the tri colours as red, green and blue, space is called as RGB colour space [16]. The colour retinal images are converted to the corresponding gray images as shown in Figure 4.
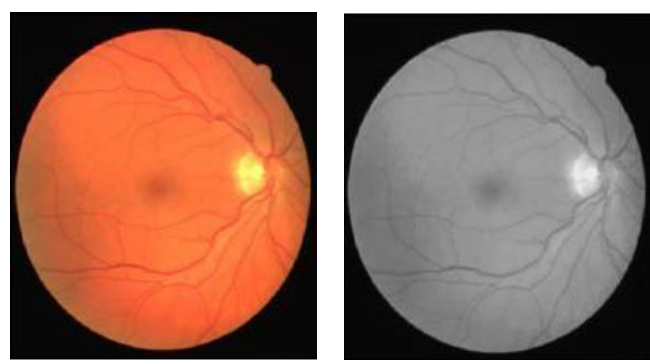

Figure 4 Original and gray level optical image 1

This is achieved by neglecting the hue and saturation and only selecting the intensity of the gray level as average of RGB spaces mathematically given as;

$$
I(:,:)=\frac{R(:,:)+G(:,:)+B(;,:)}{3}
$$

\section{HISTOGRAM EQUALIZATION}

Histogram equalization (HE) is one of the widely used methods for improving the contrast of the given images $[3,7$, and 17]. The enhanced images have a uniform distribution of the gray levels. The current section deals with the various histogram based contrast enhancement methods sequentially.

\subsection{Global Histogram Equalization}

The HE method flattens and stretches dynamic range of the image histogram thus results in overall contrast improvement $[3,4]$. Cumulative density function (CDF) is efficiently used to generate the flat histogram. The $\mathrm{CDF}$ function is obtained by simply summing up the Probability density functions (PDF) and is given as;

$$
\boldsymbol{S}=\boldsymbol{T}(\boldsymbol{r})==\int_{0}^{r} \boldsymbol{P}(\boldsymbol{r}) d r ; 0 \leq r \leq 1
$$

Now differentiating the equation (2) will give;

$$
\frac{d s}{d r}=P(r)
$$

Thus finding the CDF will flatten the histogram. But HE method may significantly change the brightness of an input image and cause problem in some applications where 
brightness preservation is necessary. Figure 5 shows an example of the original and equalized image along with their histogram.
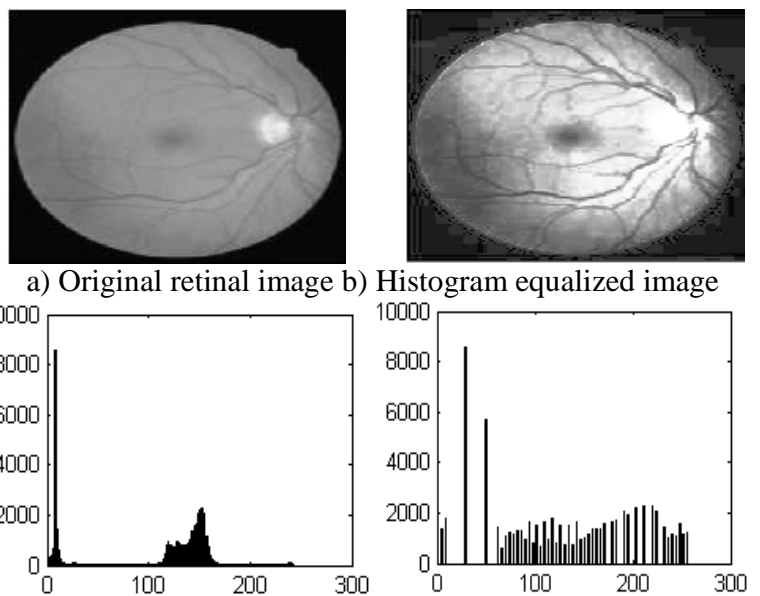

c) Original histogram

d) Equalized histogram

Figure 5 Example of retinal image enhancement

\section{THRESHOLDING BASED SEGMENTATION}

For gray images, separation of darker background from brighter foreground is thresholding. Optical images do not have such simple histograms hence thresholding require some other techniques. The coordinates of pixels with the maximum brightness are identified in the enhanced image. Then the row and columns are selected as;

Where, $f(x, y)$ is the gray level value of the equalized image. The threshold $T h$ is set to 251 in this paper. Now the segmentation is perfumed based on the selected threshold and the segmented image is given as;

$$
g(x, y)=\left\{\begin{array}{cl}
0 & \text { if } b w(x, y)<T h \\
255 & \text { if } b w(x, y) \geq T h
\end{array}\right.
$$

Where, $g(x, y)$ is a logical segmented image, then the optical disc boundary is extracted using sequential morphological operations.

\section{LOCATING OPTICAL DISC}

A boundary extraction method using morphological operators is used here to locate the disc. Boundary extraction method includes two fundamental processes Dilation and Erosion. Erosion is used to smoothen the image to erode the outer unwanted pixel in the retina. After the process of erosion some pixel in the optic disc will also get eroded so we are going for the process dilation to reconstruct the image and also it is used in the process of edge detection.

\subsection{Erosion of Threshold Image}

Erosion is a morphological operator which erodes he features of the image. Let the $\mathrm{B}$ is represented by the structuring mask and $\mathrm{X}_{\mathrm{x}, \mathrm{y}}^{\mathrm{L}}$ is the threshold image. Then the gray-scale dilation of $\mathrm{X}_{\mathrm{x}, \mathrm{y}}^{\mathrm{L}}$ by $B(x, y)$ is defined as

$$
\begin{aligned}
& \left(\mathrm{X}_{\mathrm{x}, \mathrm{y}}^{\mathrm{L}} \odot B\right)(x, y) \\
& =\min \left\{\mathrm{X}_{\mathrm{x}, \mathrm{y}}^{\mathrm{L}}\left(x-x^{\prime}, y-y^{\prime}\right)\right. \\
& \left.+B\left(x^{\prime}, y^{\prime}\right) \mid\left(x^{\prime}, y^{\prime}\right) \epsilon D_{B}\right\}
\end{aligned}
$$

Where, $D_{B}$ is the domain of the structuring element $B$ and $A(x, y)$ is assumed to be $-\infty$ outside the domain of the image and $(x, y)$ is the pixels of the image.

\subsection{Dilation of Threshold Image}

Let the $\mathrm{B}$ is represented by the structuring mask and $\mathrm{X}_{\mathrm{x}, \mathrm{y}}^{\mathrm{L}}$ is the threshold image. Then the gray-scale dilation of $\mathrm{X}_{\mathrm{x}, \mathrm{y}}^{\mathrm{L}}$ by $B(x, y)$ is defined as:

$$
\begin{aligned}
\left(\mathrm{X}_{\mathrm{x}, \mathrm{y}}^{\mathrm{L}} \oplus B\right)(x, y) & =\max \left\{\mathrm{X}_{\mathrm{x}, \mathrm{y}}^{\mathrm{L}}\left(x-x^{\prime}, y-y^{\prime}\right)\right. \\
& \left.+B\left(x^{\prime}, y^{\prime}\right) \mid\left(x^{\prime}, y^{\prime}\right) \in D_{B}\right\}
\end{aligned}
$$

Where, $D_{B}$ is the domain of the structuring element $B$ and $A(x, y)$ is assumed to be $-\infty$ outside the domain of the image and $(x, y)$ is the pixels of the image.

\subsection{Extracting the boundary of the Disc}

In order to find the inner part of the disc, subtract the eroded image from the dilated image. After the process of image subtraction we will get the edges of the optic disc.

$$
e g=\left(\mathrm{X}_{\mathrm{x}, \mathrm{y}}^{\mathrm{L}} \oplus B\right)(x, y)-\left(\mathrm{X}_{\mathrm{x}, \mathrm{y}}^{\mathrm{L}} \odot B\right)(x, y)
$$

\section{EXPERIMENTAL RESULTS}

In this paper the performance of the proposed optic disk method was evaluated based on the determined optical disk location with regard to the segmented optic disc.

\subsection{Input Images Used}

The input Retinal images used for the simulation are taken from the different data sets and references are shown in Figure 6 . The optical image 1 and 4 are the image of right eye, image 2 and 3 are the image of left eyes. Some of the images suffer from the poor contrast due to noise optical environment. For the analysis purpose all the images are resized to same image size of $240 \times 320$.

\subsection{Results of Optical Disc Segmentation}

The each segmented optic disc consists of a different canter point and a radius. For performance evaluation twenty retinal images were tested by the proposed algorithm. The results of the four distinguished images from database are presented here. Figure 7 shows the sequential results of proposed segmentation method and optical disk detection for the input optical image 1 .

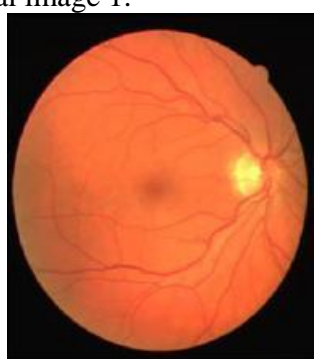

a) Optical image 1

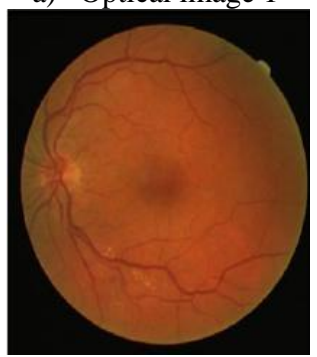

c) Optical image 1
Figure.6 Original input images used in the paper

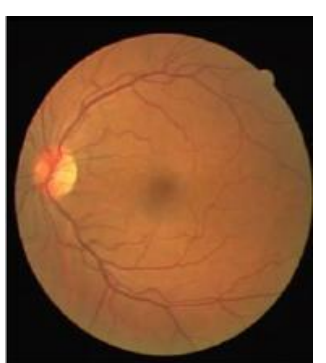

b) Optical image 2

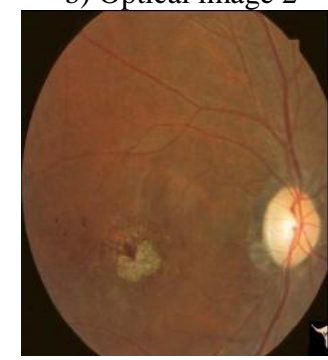

d) Optical image 4
ges used in the paper 


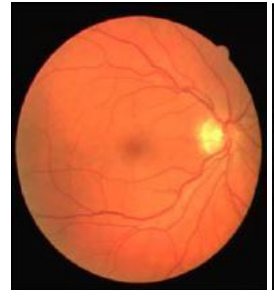

a)

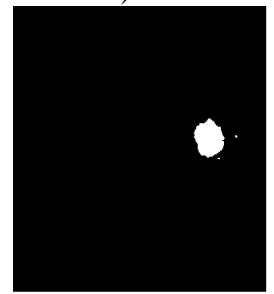

d)

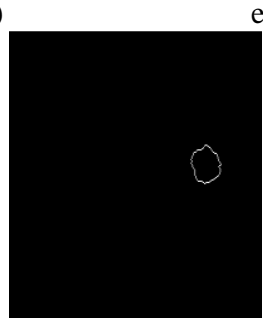

g)

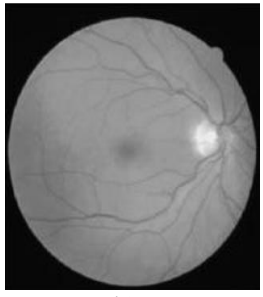

b)

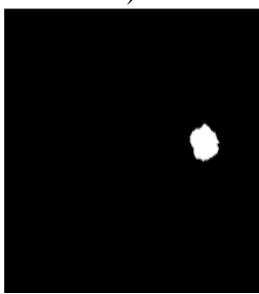

e)

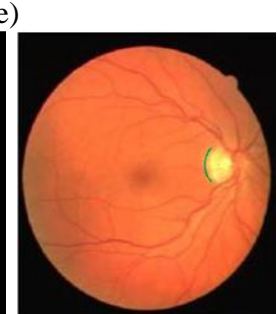

h)

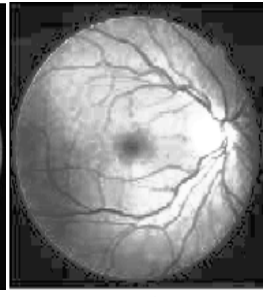

c)

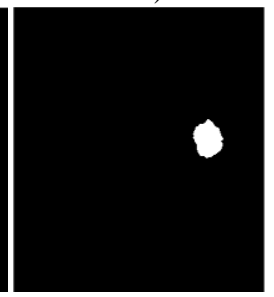

Figure 7 Sequential results of the proposed method a)

Original optical image 1, b) Gray converted image, c) histogram equalized image, d) Threshold image, e) Eroded

image, $f$ ) Dilated image, $g$ ) Difference of dilated and eroded images showing optical disc boundary, h) detected optical disc after circle fitting.

The method identifies the optical disc efficiently if the proper threshold is selected. Figure 8 presents the result of the detected optical disc for various input images after thresholding. It can be observed that method performs well for both right and left eyes images. The coordinates of canter and the radius of the detected optical disc are respectively compared in Table 1 and Table 2 for detected optical disc with and without equalization. The equalized image represents the higher disc radius thus gives better segmented disc. Also the threshold is different for different images if equalisation is not used. While after equalization same threshold may be used for most of the images. The threshold used with equalization for this paper is 251 .

Table 1 Comparison of canter

\begin{tabular}{|c|c|c|c|}
\hline $\begin{array}{c}\text { S. } \\
\text { No }\end{array}$ & Images & $\begin{array}{c}\text { Disc Canters } \\
\text { Without } \\
\text { Equalization }\end{array}$ & $\begin{array}{c}\text { Disc Canters } \\
\text { With } \\
\text { Equalization }\end{array}$ \\
\hline 1 & Optical image 1 & 250,112 & 249,111 \\
\hline 2 & Optical image 2 & 56,107 & 59,101 \\
\hline 3 & Optical image 3 & 55,129 & 57,130 \\
\hline 4 & Optical image 4 & 283,171 & 283,174 \\
\hline
\end{tabular}

Table 2 Comparison of disc Radius

\begin{tabular}{|c|c|c|c|}
\hline $\begin{array}{c}\text { S. } \\
\text { No }\end{array}$ & Images & $\begin{array}{c}\text { Disc radius } \\
\text { without } \\
\text { Equalization }\end{array}$ & $\begin{array}{c}\text { Disc radius } \\
\text { with } \\
\text { Equalization }\end{array}$ \\
\hline 1 & Optical image 1 & 15.1274 & 16.2934 \\
\hline 2 & Optical image 2 & 15.7467 & 18.9265 \\
\hline 3 & Optical image 3 & 13.7023 & 14.45632 \\
\hline 4 & Optical image 4 & 28.7590 & 30.3611 \\
\hline
\end{tabular}
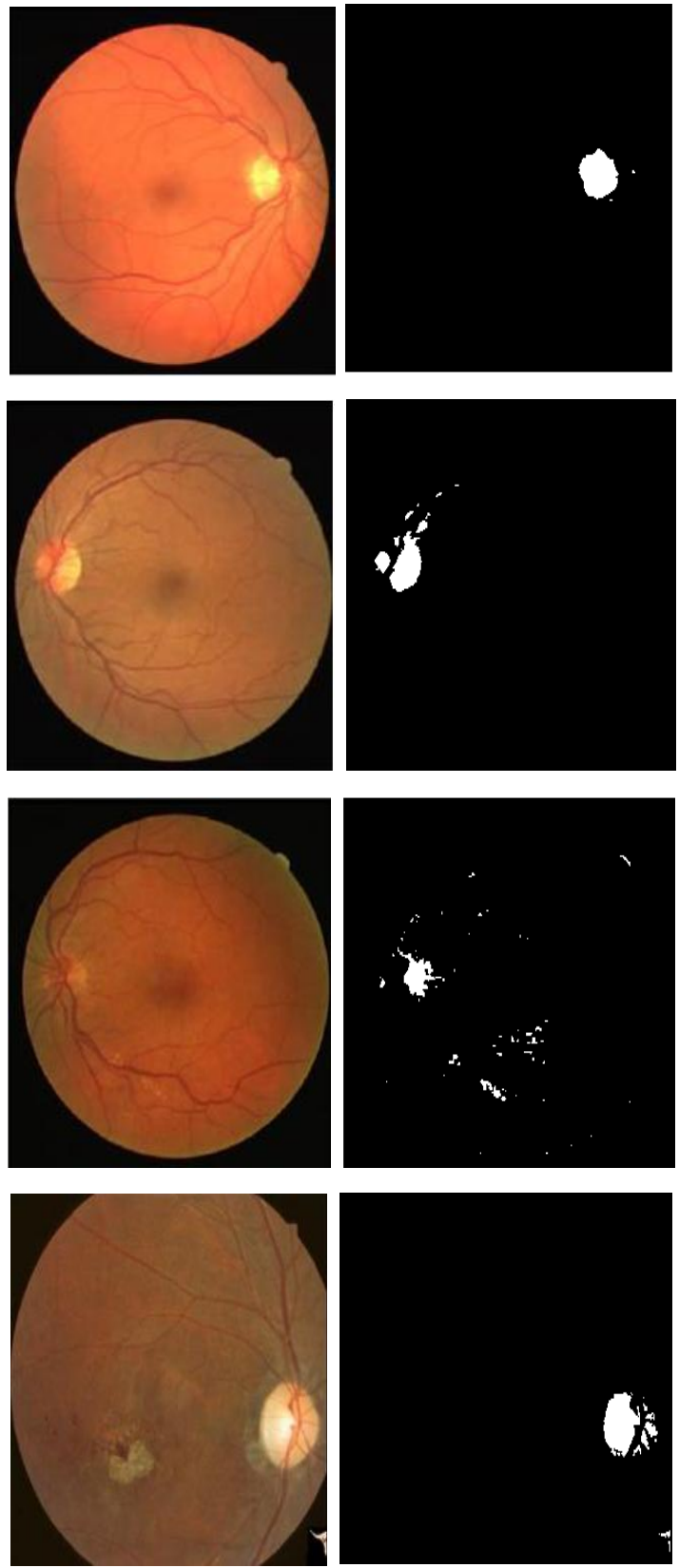

Figure 8 Results of segmented optical disc after the thresholding a) left column are input image, b) right column is threshold image

\section{CONCLUSION}

In this paper a segmentation approach for the localization of the optic disc in retinal images is presented using gray level thresholding. The method uses the morphological operators for the optical disc detection. Algorithm is currently tested on the twenty retinal images from standard databases. It is found that proposed method is fast and efficient enough for retinal image segmentation. It is observed that thresholding without enhancement is unable to identify the optical disc for low contrast images and it also need a different threshold for different retinal images. The canter of disc and disc radius are calculated and compared. It is found that enhancing the image using histogram equalization before segmentation improves the efficiency significantly. It allows us to use the same threshold for most of the images. In future the efficient circle fitting and other brightness preserving enhancement methods 
can be used to improve the efficiency of the canter point detection.

\section{ACKNOWLEDGMENTS}

Author wishes to acknowledge each and every individual who have supported for the current work directly or indirectly.

\section{REFERENCES}

[1] Adria Perez Rovira; Emanuele Trucco , "Contextual optic disc location in retinal fundus images ", Journal of Modern Optics, Volume 57, Issue 2 January 2010 , pages $136-144$

[2] A. D. Fleming, K. A. Goatman, S. Philip et al. "Automatic detection of retinal anatomy to assist diabetic retinopathy screening", Physics in Medicine and Biology 52, pp. 331345, 2007.

[3] Vasile V. Buruloin, Mihai Ciuc, Raugaraj M. Rangavyan, Loic Kjj, Constantim Vertan., "Histogram equalization of colour images using the adaptive neighbourhood approach", Proc. SPIE 3646, Nonlinear Image Processing, X, 330, 1999.

[4] Agung W. Setiawan, Tati R. Mengko, Oerip S. Santoso, Andriyan B. Suksmono, "Color Retinal image enhancement using CLAHE”, IEEE Conf. 2007

[5] Etta D, Pisano, S. Zong, R. E Jhonston "Contrast limited adaptive histogram equalization image processing to improve the detection of simulated speculation in Dense Monograms", Journal of Digital Imaging, vol. 11, No. 4, pp 193-200, 1998

[6] Huiqi Li and Opus Chutatape, "Automatic location of optical disc in retinal images" IEEE transaction on Electrical and electronic engineering. 2001, vol. 2 pp 837840

[7] P. C. Siddaligaswamy, G.K. Gautam, "Automated detection of anatomical structures in retinal images", IEEE International conf. ICCIMA 07, vol. 3, pp 164-168, 2007.

[8] A. Daskalakis, D. Cavouras, P. Bougioukos, S. Kostopoulos, P. Georgiadis, I. Kalatzis and G. Nikiforidis," "An Efficient CLAHE-based, Spot-Adaptive, image segmentation technique for improving Microarray Genes' Quantification", in 2nd International Conference on Experiments/ Process/ System Modelling/Simulation/ Optimization (IC_EpsMsO), Athens, Greece, July 4-7, 2007

[9] Muhammad Suzuri Hitam,. Wan Nural Jawahir Hj Wan Yussof, Ezmahamrul Afreen Awalludin,, Zainuddin Bachok, "Mixture Contrast Limited Adaptive Histogram Equalization for Underwater Image Enhancement", IEEE international conf. 2013.

[10] M. Abdullah-Al-Wadud, M. H. Kabir, M. A. A. Dewan, and Oksam Chae, "A dynamic histogram equalization for image contrast enhancement", IEEE Trans. Consumer Electronics, vol. 53, no. 2, pp. 593 - 600, May 2007

[11] Niemeijer M. , Ginneken B., Loog M., Abramoff M.D., "Comparative study of retinal vessel segmentation methods on a publically available database", SPIE Medical Imaging, 5370 : 648-656, 2004.

[12] Foracchia M., Grisan E., and Ruggeri A., "Detection of vessel calibre irregularities in color retinal fundus images by means of a matched filter responce", IEEE trains, on Medical. Images, Vol. 19: pp 203-210, 2000.

[13] D. Santhi, D. Manimegalai, " Location of optical disc in retinal image", IEEE International conf. ICCIMA 07, vol. 3, pp 333-338, 2007

[14] Qinghui Cao, Jianli Liu, Qiuhong Zhao., "Fast automatic optical disc localization in retinal images", IEEE Proc. of Seventh International Conference on Image and Graphics, pp. 827-831, 2013

[15] D.Jude Hemanth, J.Anitha, "Hybrid clustering method for optic disc segmentation and feature extraction in retinal images", IEEE World Congress, on Information and Communication Technologies, pp. 320-325, 2012.

[16] Gopal Datt Joshi*, Jayanthi Sivaswamy, S. R. Krishnadas, "Optic Disk and Cup Segmentation From Monocular Color Retinal Images for Glaucoma Assessment", IEEE TRANSACTIONS ON MEDICAL IMAGING, VOL. 30, NO. 6, JUNE 2011.

[17] Sandeep Kumar ,Puneet Verma, Manoj Dahiya,Aakash Gupta, "Presence Of Noise In Dualistic Sub-Image Histogram Equalization Technique of Image Enhancement", International Journal of Engineering Research \& Technology (IJERT) Vol. 1 Issue 3, May 2012.

\section{AUTHORS}

Prashant Choukikar: have completed the BE in Electronics and Communication engineering from MITS college Bhopal and is currently pursuing $M$. Tech degree from SISTECH college Bhopal India.

Prof. Arun Kumar Patel : Have received M. Tech degree and pursuing $\mathrm{PhD}$, and is currently working as Astt. Prof at SISTEC College Bhopal in Electronics and communication department

Prof. Manish Soni: Have received M. Tech degree and is currently working as Astt. Prof at SISTEC College Bhopal in Electronics and communication department

Dr. Ravi Shankar Mishra: Have received $\mathrm{PhD}$ degree in the VLSI field from the MANIT Bhopal, and is currently working as Head of the department ECE SISTEC College Bhopal India. 